\title{
Introduction of a New EMS Protocol Using the Communities of Practice Educational Model
}

\author{
Kyle A. Fratta, BS, NRP; ${ }^{1}$ Jennifer N. Fishe, MD $;^{2}$ Jennifer F. Anders, MD; ${ }^{1}$ Tessa G. Smith, \\ BA, EMT-B ${ }^{1}$
}

1. Johns Hopkins University - School of Medicine, Department of Pediatric Emergency Medicine, Baltimore, Maryland USA

2. University of Florida College of Medicine, Department of Emergency Medicine, Jacksonville, Florida USA

\section{Correspondence: \\ Kyle A. Fratta, BS, NRP \\ Johns Hopkins University - School of Medicine Department of Pediatric Emergency Medicine G1509 1800 Orleans Street \\ Baltimore, Maryland 21287 USA \\ E-mail: kafratta0602@email.campbell.edu.}

Conflicts of interest: none

Keywords: education; Emergency Medical Services; protocol

Abbreviation:

EMS: Emergency Medical Services

Received: September 4, 2018

Accepted: October 30, 2018

Online publication: January 11, 2019

doi:10.1017/S1049023X18001267

\begin{abstract}
Emergency Medical Services (EMS) protocol implementation can be a challenging endeavor given the large and diverse provider workforce. These efforts can be even more challenging given training restrictions, career and volunteer combination EMS agencies, and inconsistent work schedules. In an effort to educate as many providers as possible in a relatively short time, the community of practice educational model was used during a new evidence-based EMS protocol implementation. This model identifies providers who are enthusiastic during initial training as advocates. These advocates then continue to educate their peers going forward. This allows for the initial educational effort to continue to propagate during pilot testing and beyond. During this protocol implementation, a total of 17 educational visits were made to EMS stations and 43 providers were identified as advocates.
\end{abstract}

Fratta KA, Fishe JN, Anders JF, Smith TG. Introduction of a new EMS protocol using the communities of practice educational model. Prehosp Disaster Med. 2019;34 (1):108-109.

The PDTree is an evidence-based protocol to guide Emergency Medical Services' (EMS) pediatric destination decisions. The PDTree was developed by an expert panel using an iterative, modified-Delphi approach. It is currently being pilot tested in three diverse Maryland (USA) EMS agencies: urban Baltimore City, which contains a unionized career workforce; suburban Prince George's County, which is a mixed career/volunteer workforce with over 40 stations; and rural Queen Anne's County, which is a mixed career/volunteer workforce and operates in a county without a hospital. Our group utilized the community of practice social learning model for the educational, introductory component of the PDTree's implementation.

Communities of practice are groups of individuals who interact together based on a common purpose to disseminate knowledge and innovation and allow for collaboration over a long period of time. ${ }^{1}$ In order to establish this community, PDTree team members identified providers who could serve as advocates and continue to educate others on the protocol's use after the initial on-site training. Using this approach allowed us to connect personally with a larger number of individual providers, and to be aware of and respond to provider educational needs during pilot testing.

The curriculum was multi-faceted to cater to the large and diverse provider workforce we were tasked with training. At the forefront of our protocol rollout was traditional classroom education utilizing a Microsoft PowerPoint (Microsoft Corp.; Redmond, Washington USA) presentation. This presentation included a review of the evidence, description of the tool, and discussion of the pediatric capabilities of the local hospitals. At the end of presentation, open-ended clinical questions asked providers to utilize the PDTree to determine the destination hospital for varied pediatric clinical scenarios. The PowerPoint included voiceovers using carefully phrased scripts asking providers "Where would you triage this child to?" rather than "Where does the PDTree instruct you to triage this child?"

Following the dissemination of the PowerPoint module, the PDTree trainers visited individual fire stations to engage the providers while on-duty. The PDTree trainers themselves were prehospital professionals who could relate to providers' viewpoints and needs. At those training sessions, individuals were identified to act as advocates and continued contacts for the team for the purposes of further dissemination, advocacy, continued 
education, and feedback. These advocates were given access to training and reference materials and were encouraged to hold both formal and informal education for their fellow providers over the coming weeks and months. Additionally, an off-site session was recorded and uploaded to online training portals for each agency to allow for improved access to the training materials.

Due to union restrictions, station visits were not feasible in Baltimore City. Therefore, we hosted several traditional classes during re-certification classes at the Baltimore City centralized training center. Additionally, we hosted a "train-the-trainer" session in an attempt to cultivate a group of advocates at one time who could go forward and train their co-workers as permitted.

A total of 17 educational visits were made to EMS stations between May 25, 2017 and June 26, 2018 in addition to the posted online training. During these visits, 43 EMS providers were identified as PDTree advocates. The PDTree protocol took effect July 1, 2018 in our three pilot EMS agencies. The pilot program will run for one year with data assessment every three months. The major assessment of the educational initiative will be proper triage and destination decision of pediatric patients transported by EMS.

Use of the communities of practice educational model is an ideal tool for EMS provider education and protocol implementation. Using this model allowed our team to educate a large provider workforce working on rotating and inconsistent schedules spread over a large geographic area in a relatively short time. The differences in work schedules, career and volunteer EMS agency training requirements, and union restrictions would have made a traditional blanket educational initiative unmanageable. All EMS medical directors, fellows, and other agency leaders should consider adoption of the community of practice learning model for education and implementation efforts.

Reference

1. Wenger E. Communities of Practice: Learning, Meaning, and Identity. Cambridge, United Kingdom: Cambridge University Press; 1998. 